\title{
Life at the Duke of Singapore
}

uke University, Durham, North Carolina, USA, and the National University of Singapore (NUS) formally formed a partnership in 2005 to establish a new medical school (Duke-NUS Graduate Medical School, herein referred to as Duke-NUS) (Figure 1). The school is based in Singapore and, while it is formally part of the NUS system, it is distinct in that it has an independent governing board that includes representatives of Duke University. The students, the first of whom matriculated in August 2007, learn from a curriculum based on that of Duke University School of Medicine and upon successful completion of the course, they will be awarded a joint Doctor of Medicine (MD) degree from both Duke University and the NUS.

Other links to Duke University have been established through the administrative team, faculty, and staff; for example, Patrick Casey (Figure 2), the senior vice dean of research at Duke-NUS, spends most of his time in Singapore but also remains on the faculty at Duke University, where he is the James B. Duke Professor of Pharmacology and Cancer Biology and runs a highly active laboratory. Casey took some time to talk to the JCI about Duke-NUS and the international partnership underpinning it.

JCI: Creating Duke-NUS took several years, at what point in the process did you first get involved?

Casey: I became involved in 2003, just after the signing of the Memorandum of Understanding between Duke University and the Singapore government, outlining the desire to develop a new research-oriented medical school with a curriculum and culture paralleling that of the Duke University School of Medicine.

JCI: What motivated you to take the position as senior vice dean of research at Duke-NUS?

I was initially asked by the dean, R. Sanders (Sandy) Williams, who ultimately became the founding dean of Duke-NUS, to join a planning committee to advise on just how Duke might actually be able to pull this off. My area of focus was to have been the basic/translational science component of the initiative but that morphed into much more later, as for the first two years, I was the senior Duke person on the ground in Singapore. I made trips to Singapore with various Duke representatives in late 2003 and mid-2004 and by then was quite enamored with the possibilities and potential of the enterprise. By the time of the signing of the formal agreement in early 2005, both my wife, Mei Wang, and I saw a number of advantages to becoming the founding Duke investigators in the school. Mei is a MD/PhD who had left research behind in the mid-1990s to pursue medicine full-time. She had a desire to move back into research, but that would have been quite difficult in the current US climate for someone who had been a fulltime clinician for so long, so this gave her an opportunity as well as myself; I had a desire to build something special without having to give up my own personal research program. The opportunity to have our children spend time in Asia also factored in; they were two and ten at the time and not

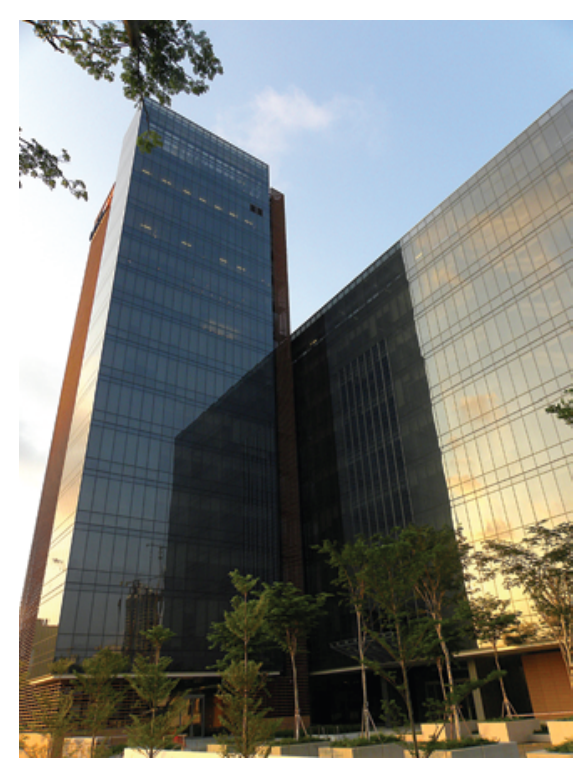

\section{Figure 1}

The Khoo Teck Puat Building at Duke-NUS, a medical school established in Singapore by both Duke University and the NUS. Image provided by Frank Starmer, Associate Dean of Learning Technologies, Duke-NUS.

feeling particularly Amerasian growing up in North Carolina. All in all, it seemed like a great opportunity for the whole family, as we all got to participate in something that we felt (or at least hoped) would be quite special. We have not been disappointed. JCI: What do you anticipate will be your biggest challenge in this position?

Casey: The biggest challenges I felt Duke University would face were three-fold. First, would we be able to recruit top-notch faculty to Singapore? We had decided very early on that if this were not to be a Dukequality entity across the board, we did not want to undertake it. Second, would we be able to attract Duke-quality students into the medical school and eventually into PhD programs? Third, would we be able to manage the expectations of all the stakeholders? This entire initiative had six such stakeholders: three Singapore government ministries (Education, Health, and Trade and Industry), NUS, Singapore Health Services (SingHealth, one of the two major health care delivery entities in Singapore), and Duke University. Each of these stakeholders had a somewhat different view of what the Duke-NUS Medical School should be, and it made for some very interesting times in the first few years!

JCI: Has the funding crisis in the US affected the development of Duke-NUS?

Casey: I would have to say that the US's loss has been Singapore's gain. It has certainly not hurt us in recruiting faculty, and support for biomedical sciences has continued to increase in Singapore despite the global economic down turn.

JCI: What are the biggest differences between medical training at the Duke-NUS and most US institutions?

Casey: In the preclinical arena, the biggest difference is that we use a style of education termed "Team-Based Learning," in which students do not go to formal lectures, rather they assimilate knowledge from downloaded lectures from Duke and from various reading sources and assemble in teams to work through problems under the eye of basic science and clinical faculty. It is quite an amazing system and would take awhile to explain fully. It started in the US, at Wright State University, Dayton, Ohio, and was further developed by the two vice deans of medical education, Bob Kamei (Duke-NUS) and Ed Buckley (Duke). In the clinical years, the training is quite similar except that it has taken some time and effort to educate clinicians in our major teaching hospitals (Singapore General Hospital and KK Women's and Children's Hospital) in the US style of an interactive student-faculty relationship, where 


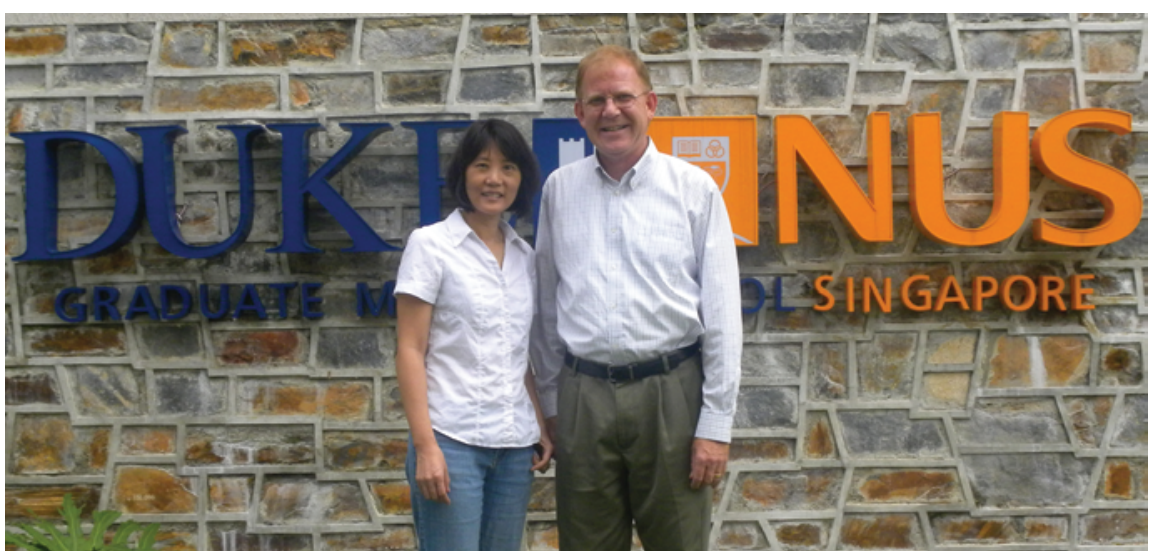

Figure 2

Patrick Casey and his wife Mei Wang have research programs at Duke-NUS and are enjoying the opportunities afforded to them by life in Singapore.

the students form an integral part of the clinical care delivery teams in the hospitals. There are a few substantive differences in the training related to disease prevalence, of course. This is especially true in the area of infectious diseases (for example, dengue is major problem here but rarely encountered in the US) and in cancer, where liver, gastric, and nasopharyngeal cancers are much more prevalent in Asia than the US.

JCI: What are the cultural differences between education and research in Singapore versus Durham, and how have they affected the Medical School?

Casey: The one we were most concerned with before we came over never actually materialized. Specifically, we were afraid that the stereotype Asian student mentality, wherein students never challenge faculty and are instead simply passive learners, even when they feel an authority figure may not be fully up to speed, so to speak, would impair our ability to deliver the quality of experience we felt necessary for the training of inquiry-based physicians and physicianscientists. We have been quite pleased with how this has turned out, as our students are not shy about challenging the faculty! Much of this has to do with the fact that so many of them, even many from Singapore and its neighboring countries, did their undergraduate training in the US, Australia, or the United Kingdom. Also, the culture of our major "feeder" school, NUS, has changed markedly in the past decade, such that they do more to promote the inquisitive/challenge-type attitude in students. Not all local faculty, particularly in the clinical arena, are completely accepting/appreciative of this as yet, but they are adapting.
JCI: The first medical students began class in August 2007. How have the first two years gone?

Casey: Incredibly well; as well as we could have hoped, in fact. That is not to say there have been no hiccups, but they are a truly inspired and inspiring group of students and a joy to work with.

JCI: How are the Singapore students different for those you taught at Duke?

Casey: Personally, I see very little difference. That said, I am actually masquerading as a medical school instructor, as essentially all my experience in the education arena, prior to coming over, was in graduate school programs.

JCI: Practically, how much interaction has there been between the medical students and researchers at Duke-NUS and those at Duke?

Casey: We have six faculty at Duke-NUS who moved over fully from Duke, including the current Dean K. Ranga Krishnan and another 15 Duke faculty who spend at least two months a year in Singapore, so there is a tangible Duke presence on the DukeNUS campus at all times. In addition, we have an exchange program whereby $2-3$ Duke students spend their third year (in the Duke curriculum, all MD students spend their third year in research) in Singapore, and 2-3 Duke-NUS students spend the year in Durham. In addition, there is a constant flow of both educators and scientists between the two campuses; it is rare that a week goes by and I do not encounter someone from Duke who happens to be in Singapore that week.

JCI: Do you feel that you are training Singapore students for the same types of careers for which you were training American students?

Casey: No question, and this is why Singapore went to such effort and expense to create this second medical school. They are investing heavily in the biomedical sciences, with the plan that it will become a major pillar of their economy, which is clearly already happening. Duke-NUS aims, as does Duke, to train physicians and physician-scientists who will play teading roles in determining the future of all aspects of medicine from translational medicine, to innovative clinical care, to inventive changes in how health care is delivered, and to major roles in the emerging biotech/ pharma industries.

JCI: What is your vision of the future for Duke-NUS?

Casey: Our goal is nothing less than to be considered the top medical school in Asia within the next decade and to be viewed similarly to a top-ten medical school in the US. We also aim to be considered one of the premier research institutions in the world, particularly in the area of translational medicine. The five Signature Research Programs we have created (analogous to departments in a traditional medical school, but each spans basic to clinical sciences) are Cancer and Stem Cell Biology, Neuroscience and Behavioral Disorders, Cardiovascular and Metabolic Diseases, Emerging Infectious Diseases, and Health Services Research, and we anticipate that each of the programs will contribute unique and innovative findings and technologies to their respective fields.

JCI: Based on your first years involved in education in Singapore, are there any lessons you have learned that you could bring back to the US to improve our education or research?

Casey: I am completely sold on the TeamBased Learning approach to the preclinical training for MD students, and we plan to incorporate this methodology significantly into the $\mathrm{PhD}$ program in Integrative Biology and Medicine that we are currently establishing. It is truly amazing how the method changes education from a teaching experience to a true learning experience for both the students and the faculty. Also, the idea of developing programs that span basic to clinical research, rather than traditional, separate departments in the basic and clinical sciences, we feel will enhance the success in undertaking translational medicine initiatives.

\section{Karen Honey}

